\title{
Multi-spectral imaging for Thermochromic Liquid Crystal based Particle Image Thermometry: A proof of concept
}

\author{
T. Käufer ${ }^{1 *}$, S. Moller ${ }^{1}$, M.Rosenberger ${ }^{2}$, G. Notni ${ }^{2}$, C. Cierpka ${ }^{1}$ \\ ${ }^{1}$ Technische Universität Ilmenau, Institute for Thermodynamics and Fluid Mechanics, 98693 Ilmenau, \\ Germany \\ ${ }^{2}$ Technische Universität Ilmenau, Group for Quality Assurance and Industrial Image Processing, 98693 \\ Ilmenau, Germany \\ *theo.kaeufer@tu-ilmenau.de
}

\begin{abstract}
In this contribution, a novel imaging approach for Thermochromic Liquid Crystal (TLC) based Particle Image Thermometry (PIT) is demonstrated. In contrast to state of the art approaches, a multi-spectral camera was used to record the color response of the Thermochromic Liquid Crystals seeding particles. An experiment with a transparent, water-filled, cylindrical cell as the central element was set up to investigate the novel approach. The temperature in the cell can be controlled by adjusting the temperature of the bottom and top plate. Calibration images at eleven different temperatures ranging from $18^{\circ} \mathrm{C}$ to $21.6^{\circ} \mathrm{C}$, as well as images of a stable thermal stratification, were recorded. 90 percent of the calibration data was used to train a neural network $(\mathrm{NN})$ to predict the temperature. The remaining 10 percent of the calibration data and the data of the stable thermal stratification were used to test the NN. The tests show that the deviation between predicted and ground truth temperature is mostly below $0.1 \mathrm{~K}$ and that the linear profile of the stable thermal stratification can be predicted with a maximum deviation of $\approx 0.15 \mathrm{~K}$. This shows that multi-spectral imaging with neural networks for data processing is feasible and a promising concept.
\end{abstract}

\section{Introduction}

To fully describe temperature-driven flows, quantitative knowledge of both velocity and temperature is of vital importance, which requires the measurement of both quantities. To measure fluid velocities, Particle Image Velocimetry (PIV) and Particle Tracking Velocimetry (PTV) are well-established, sophisticated methods and are the go-to techniques for many experimental investigations when an optical access can be ascertained. Likewise, for optical temperature measurements in fluids, several different methods have been developed and are applied depending on the field of application, expected temperature range, and required uncertainty. One approach is called Laser Induced Fluorescence (LIF). To perform LIF, one or two temperature-sensitive dyes are added to the fluid. Those dyes are then excited by a laser, and the fluorescence emission spectrum is observed from which the temperature can be derived. However, those dyes result in a slight opacity of the fluid of investigation, which decreases the signal-to-noise ratio of the particle images if particles are added for simultaneous PIV measurements. In this case, the seeding particles required for the PIV measurements also interfere with the temperature measurements, see Funatani et al. (2004). An alternative approach is to use temperature-sensitive seeding particles for temperature measurement. The major advantage is that the seeding particles can often also be employed for the velocity measurements, and, therefore, both measurements do not interfere with each other. Those temperature-sensitive particles may, for instance, have temperature-related luminescent (Massing et al. (2016)), phosphorescent (Abram et al. (2018)) or reflective properties (Dabiri and Gharib (1991)). This technique is called Particle Image Thermometry (PIT).

Commonly used seeding particles with temperature-related reflective properties are encapsulated Thermochromic Liquid Crystals (TLCs). When TLCs are illuminated by white light, the reflected wavelength range and thus the color shade of the TLCs depends on the temperature and the observation angle. 
The combination of PIV and PIT has proven to be well-suited for the experimental investigation of heat and momentum transfer in large aspect ratio Rayleigh-Bénard convection where the temperature range to be considered is oftentimes moderate, see Moller et al. (2021). For this application, uncertainties in the temperature measurements of $0.1 \mathrm{~K}$ and less are reported by Moller et al. (2019).

The current state of the art is to record the color response of the TLCs with an RGB (Red, Green, Blue) color camera. After recording the RGB-images, the color information is then transformed into the HSV/I (Hue, Saturation, Value/Intensity) color space because the perceptible change in color appearance can be sufficiently described by the Hue value only, which means that a bijective relation between Hue value and temperature exists. However, neural networks (NN) were also successfully applied to estimate the temperature from the intensity data, see Anders et al. (2020).

The novelty of this work is the application of a multi-spectral camera instead of a conventional RGB color camera to record the color response of the TLCs. In general, multi-spectral cameras feature numerous different color channels with oftentimes smaller bandwidths. Among other applications, multi-spectral cameras have already been successfully used for food quality assurance, see Rosenberger and Celestre (2016). When applied to capture the color response of the TLCs, the additional color channels allow for a more detailed spectral resolution of the TLCs compared to the three color channels of the RGB camera. Furthermore, the multiple spectral bands might also allow capturing the reflection in the near-infrared (NIR) range. This might increase the measurement range since it was shown by König et al. (2019) that the TLCs reflect slightly in the NIR range when the illumination spectrum also contains those wavelengths. Hence, the scope of this work is to demonstrate the feasibility of multi-spectral imaging for PIT.

\section{Experimental setup}

In order to test, the suitability of a multi-spectral camera for PIT an experiment was set up. A schematic top view of the setup can be seen in Figure 1(a), The central unit of the experiment is a water-filled, cylindrical cell with a height of $h=55 \mathrm{~mm}$ and an inner diameter of $d=110 \mathrm{~mm}$, which is enclosed by two plates made of aluminum, as it can be seen in Figure 1(b). The temperature of the bottom and top plate can be adjusted

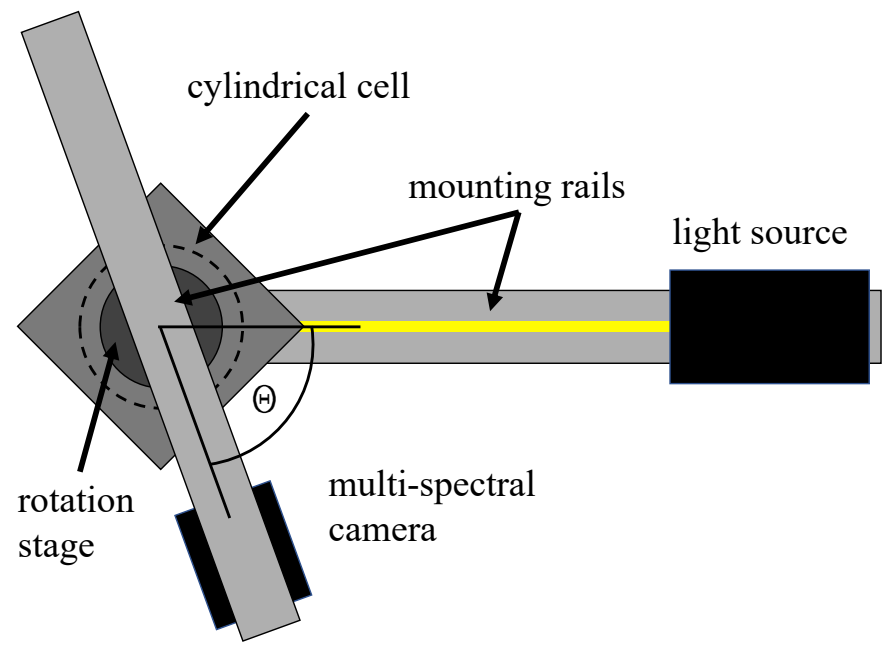

(a)

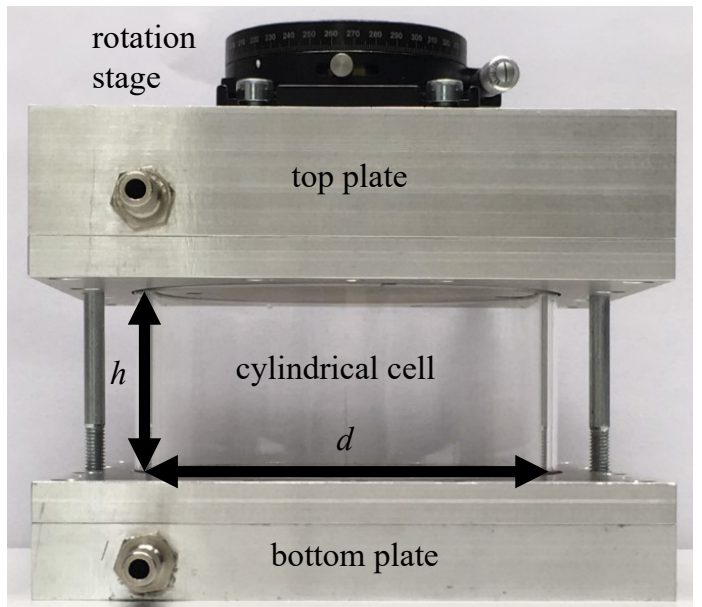

(b)

Figure 1: (a) Schematic top view on the experimental setup (a). $\Theta$ denotes the observation angle. (b) Side view on the cylindrical cell with mounted rotation stage. $h$ and $d$ denote the height and the inner diameter of the cell, respectively.

independently and can be measured by PT-100 temperature sensors. On top of the cell a rotation stage is fixed on which a mounting rail with the multi-spectral camera is mounted. Thereby, the observation angle $\Theta$ between camera and light source can be adjusted.

The used multi-spectral camera is a filter wheel camera with twelve different color channels and a sensor resolution of 1024 pixel $\times 1160$ pixel. A camera objective with a fixed focal length of $f=6 \mathrm{~mm}$ is mounted in front of the camera. For details on the camera the reader is referred to Rosenberger and Celestre (2016). The advantage of such a filter wheel is that the spectral bands of the color channels can be easily adjusted 
by exchanging the filters in the filter wheel. Yet, the usage of a filter wheel has the disadvantage that the color channels are not recorded instantaneously but subsequently, in contrast to sensor-mounted filters. This restricts the range of application of a filter wheel camera to slowly changing or stationary temperature distributions. However, the scope of this work is to investigate the feasibility of multi-spectral imaging for PIT rather than the quantification of a specific flow. Therefore, the advantage due to the customizablitiy of the color channels surpasses the disadvantage of the non-simultaneous recording.

As the light source an LED array with integrated light sheet optics is used, that generates a light sheet with a thickness of $\approx 2.5 \mathrm{~mm}$. The spectrum of the light source is depicted Figure 2, where the relative intensity $I_{\mathrm{R}}=I / I_{\max }$ is shown in dependency of the wavelength $\lambda$. One can see that the illumination spectrum of

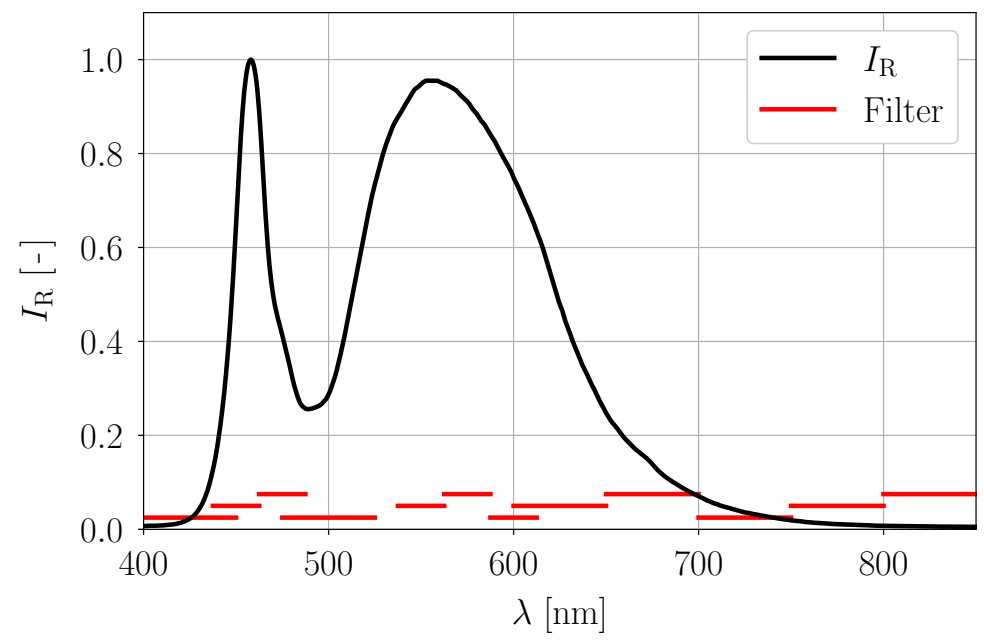

Figure 2: Illumination spectrum of the used light source. The optical filters used in the multi-spectral camera are shown as red lines in the plot. For a better visualization the vertical position of the lines is altered.

the LED ranges from a wavelength $\lambda \approx 400 \mathrm{~nm}$ to $\lambda \approx 800 \mathrm{~nm}$ with two intensity peaks at $\lambda \approx 460 \mathrm{~nm}$ and $\lambda \approx 560 \mathrm{~nm}$. The red lines in the plot depict the filter selection mounted in the filter wheel of the camera. In Table 1 the central wavelengths $\lambda_{\mathrm{c}}$ and the bandwidths $\Delta \lambda$ of the filters are listed. The bandwidth is defined as FulT Width at Half Maximum (FWHM), which means that the transmission at the edges of the bandwidth is half of the maximum transmission. The bandwidth $\Delta \lambda$ is centered around the central wavelength $\lambda_{\mathrm{c}}$ of the filter.

As seeding particles encapsulated TLCs of type R20C20W (LCR Hallcrest) were used. There nominal

Table 1: This table shows the central wavelength $\lambda_{\mathrm{c}}$ and bandwidth $\Delta \lambda$ of the used filters. The filters are numbered ascending according to their central wavelength.

\begin{tabular}{l||l|l|l|l|l|l|l|l|l|l|l|l} 
& 1 & 2 & 3 & 4 & 5 & 6 & 7 & 8 & 9 & 10 & 11 & 12 \\
\hline \hline$\lambda_{\mathrm{c}}[\mathrm{nm}]$ & 425 & 450 & 475 & 500 & 550 & 575 & 600 & 625 & 675 & 725 & 775 & 825 \\
\hline$\Delta \lambda[\mathrm{nm}]$ & 50 & 25 & 25 & 50 & 25 & 25 & 25 & 50 & 50 & 50 & 50 & 50
\end{tabular}

temperature sensitivity starts at $20^{\circ} \mathrm{C}$ and ends at $40^{\circ} \mathrm{C}$ for an observation angle of $\Theta=0^{\circ}$. However, for larger observation angles close to $\Theta=90^{\circ}$, which is often beneficial for the investigation of fluid flows with optical measuring techniques, the sensitivity is increased while the temperature range is drastically decreased, as shown by Moller et al. (2019). 


\section{Results}

After setting up the experiment, calibration measurements were performed. The goal of the calibration measurements is to determine the relationship between the intensity distribution across the color channels and the related temperature. Therefore, several isothermal states have been set inside the cell at eleven different temperature levels ranging from $18{ }^{\circ} \mathrm{C}$ to $21.6{ }^{\circ} \mathrm{C}$ by adjusting and measuring the temperature of the bottom and top plate of the cell. When the temperature sufficiently converged, a total of ten calibration sets were recorded for each individual calibration temperature. A set consists of twelve images, on for each filter. However, for further processing, only the ten filters with the central wavelengths in the range $425 \mathrm{~nm} \leq \lambda_{\mathrm{c}} \leq 725 \mathrm{~nm}$ were used since the absolute intensities for the filters $\lambda_{\mathrm{c}}=775 \mathrm{~nm}$ and $\lambda_{\mathrm{c}}=825 \mathrm{~nm}$ were too low to be meaningful. This is a consequence of the low emission of the light source at $\lambda \approx 800 \mathrm{~nm}$. Figure 3 shows the filter-wise relative intensity $I_{\mathrm{f}, \mathrm{R}}=I_{\mathrm{f}} / I_{\mathrm{f}, \max }$ for the ten filters with the central wavelengths in the range $425 \mathrm{~nm} \leq \lambda_{\mathrm{c}} \leq 725 \mathrm{~nm}$ in dependency of the temperature level. The images were recorded

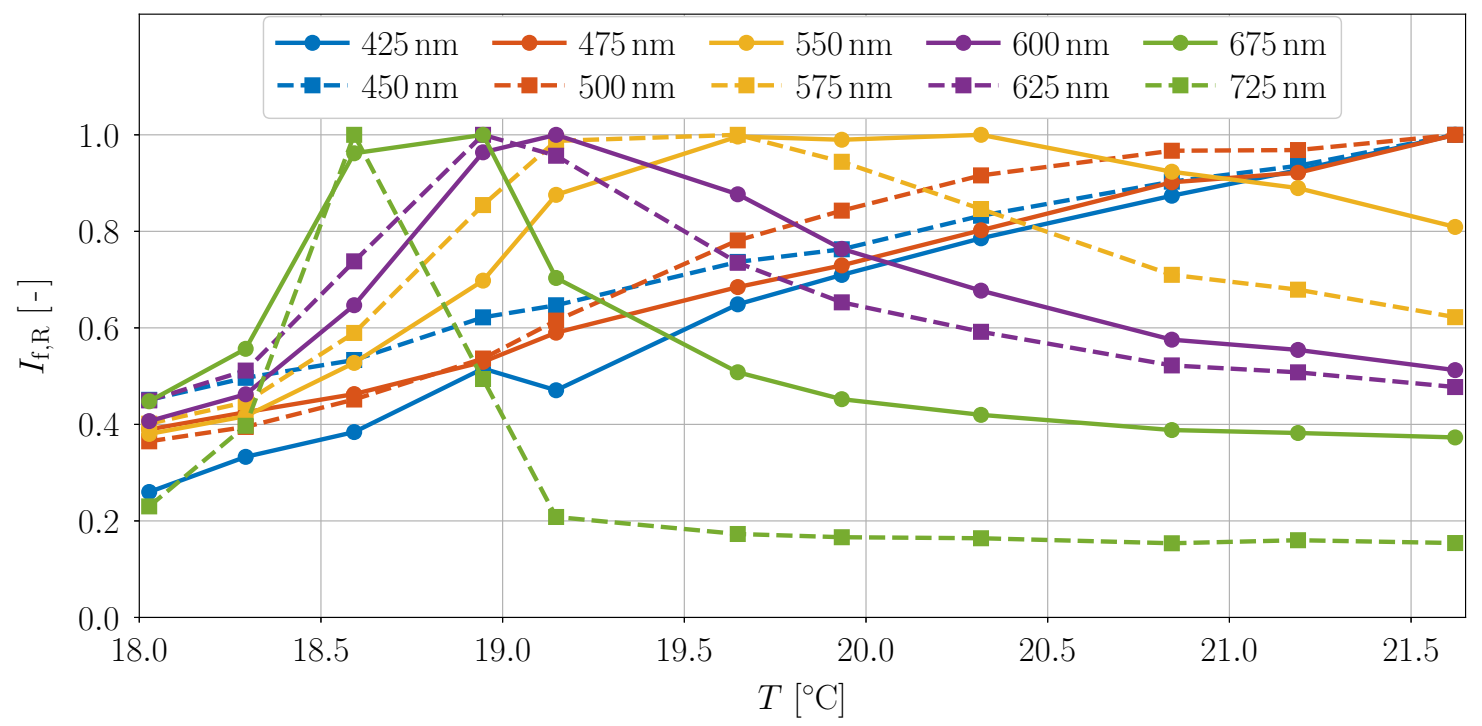

Figure 3: Transition of the filter-wise relative intensity $I_{\mathrm{f}, \mathrm{R}}$ across the calibration temperature range

under an observation angle $\Theta=70^{\circ}$ and the filter-wise intensities $I_{\mathrm{f}}$ were estimated by spatially averaging the intensities in a region that covers almost the full height of the cell but only a slim horizontal span at the center of the cell. Thereby the change of the observation angle $\Theta$ along the width and optical distortion caused by the cylindrical wall can be neglected. In vertical direction $\Theta$ is constant. For a more extensive explanation, the reader is referred to the publication of Moller et al. (2019). One can see that the relative intensities for all filters are small for the lowest temperature levels and that they rise until the curves reach their individual peak. The peak temperature of the filters is inversely related to the central wavelength of the filters. This means that the TLCs appear red for lower temperatures and continuously change their appearance through the visible spectrum until they appear blue at temperatures above $21.5^{\circ} \mathrm{C}$. By comparing the nominal starting point and the range of the TLC (start at $20^{\circ} \mathrm{C}$, range of $20 \mathrm{~K}$ ) with the filter-wise relative intensities, it gets apparent that the nominal range can only be used as a qualitative indicator. Thus, due to the influence of the illumination spectrum and the observation angle on the color appearance of the TLCs a calibration is required for every specific measurement arrangement. Furthermore, the calibration has to be performed locally since the change of the observation angle across the field of view can not be neglected in this arrangement. Therefore, the images are sliced into interrogation windows with a size of 32 pixel $\times 32$ pixel that overlap by 16 pixel.

Due to the additional data provided by the multi-spectral camera, a Hue-based calibration approach can not be easily applied. It could furthermore negate possible benefits through the increased spectral resolution. Hence, a neural network was trained to estimate the temperature from the intensity data. The NN and the data processing pipeline were implemented in the Python programming language. The NN is based on the Multi Layer Perceptron (MLP) regressor provided by the scikit-learn software library (Pedregosa et al. (2011)) and is designed as a feedforward NN. In the first step, the calibration images of the ten filters with 
the lowest central wavelength were cropped to the region of interest depicted in Figure 4 , then the images were sliced into interrogation windows and the mean intensity value of each interrogation window was calculated. Based on the intensity data and the coordinates of the center of interrogation windows, a buffer was

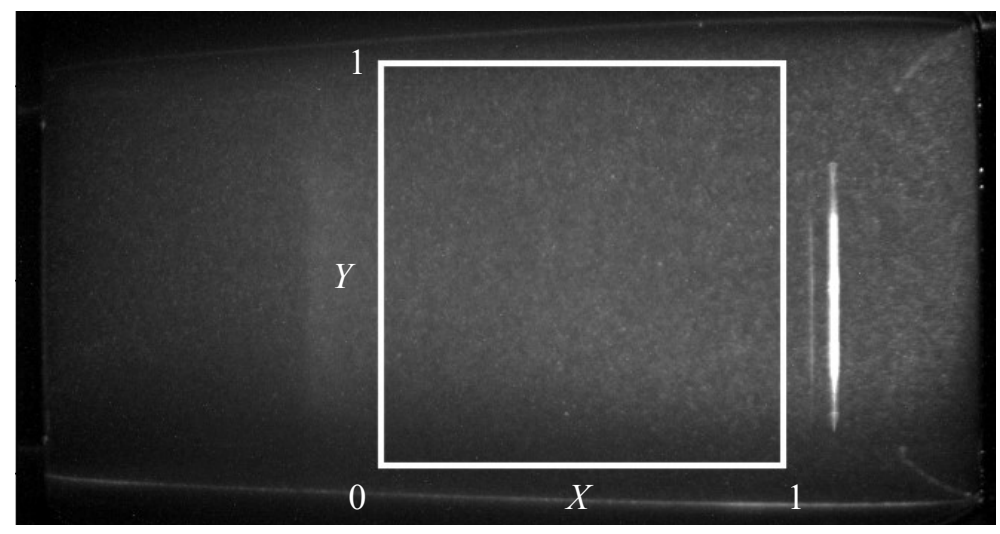

Figure 4: Exemplary image of the stable thermal stratification recorded by the multi-spectral camera through the filter with $\lambda_{c}=550 \mathrm{~nm}$. The white box denotes the region of interest. $X$ and $Y$ denote the coordinate system used to visualize the results of the NN.

created. By dividing the intensity data of each filter by the maximum occurring intensity of that specific filter, the intensity data were normalized. Likewise, the interrogation window coordinates were normalized by the size of the region of interest. Thereby all values are in the interval $[0,1]$ and had a similar scale which is beneficial for the NN. From this buffer, the data of nine sets were used for training and one set for testing purposes. The NN consists of the input layer that takes twelve inputs (ten intensities, two coordinates), six densely connected layers with 100 neurons each, and the "ReLU" activation function and an output layer with a single neuron. For the optimization, the "Adam" optimizer was used. The NN configuration was optimized experimentally. For the final training, a split of ten percent of the training data was used to validate the progress during the training and to avoid overfitting, and the $\mathrm{NN}$ was trained for 44 iterations until both training and validation loss had converged sufficiently.

To test the quality of the NN, a randomly sampled temperature distribution was used. This randomly sam-

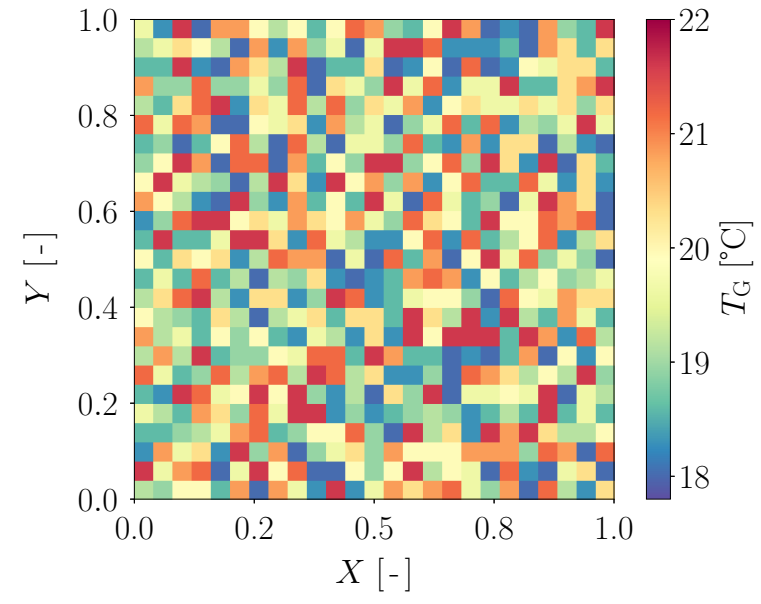

(a)

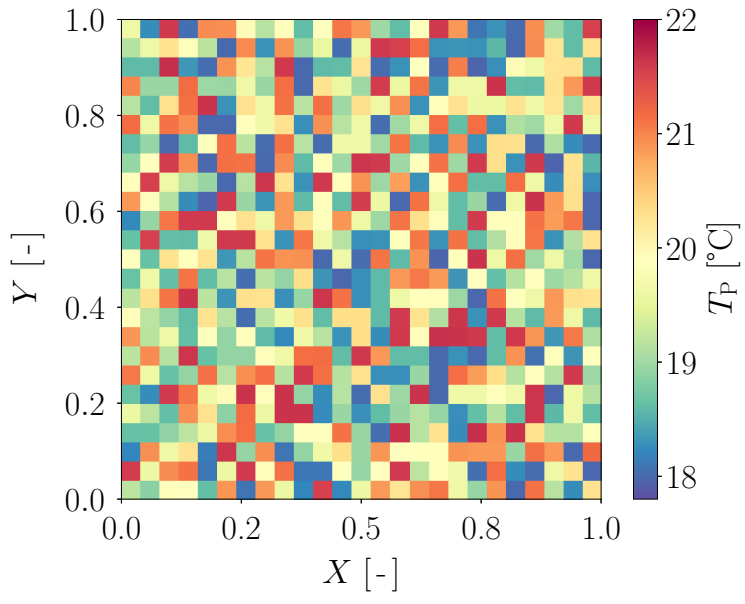

(b)

Figure 5: (a) Temperature field displaying the ground truth temperature $T_{\mathrm{G}}$ of the randomly sampled temperature distribution. (b) Temperature field showing the temperature predicted by the neural network $T_{\mathrm{P}}$. The $X$ and $Y$ coordinates are the same as shown in Figure 4.

pled temperature distribution was created from the calibration data reserved for testing purposes. For each 
interrogation window, the intensity data was randomly selected from the eleven measured calibration temperatures. The corresponding field of the ground truth temperature $T_{\mathrm{G}}$ is shown in Figure 5(a), Even though a temperature distribution like this is only of theoretical nature, it can be considered as a worst-case scenario to test if the NN is capable of predicting the temperature of each interrogation window independently, which is vital for the investigation of the temperature in fluid flows. The field of the predicted temperature $T_{\mathrm{P}}$ is depicted in Figure 5(b) By comparing the predicted temperature field and the ground truth temperature field, one can already see the quality of the prediction and that the $\mathrm{NN}$ is capable of predicting the temperature of each interrogation window independently. A more detailed view on the deviations $T_{\mathrm{P}}-T_{\mathrm{G}}$ is provided by the deviation field in Figure 6(a) and the related histogram in Figure 6(b). One can see that the deviation is small

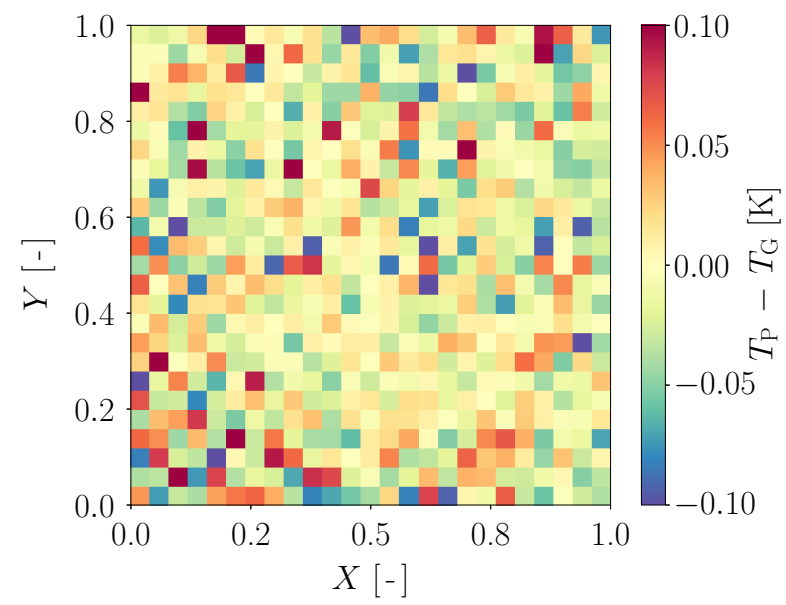

(a)

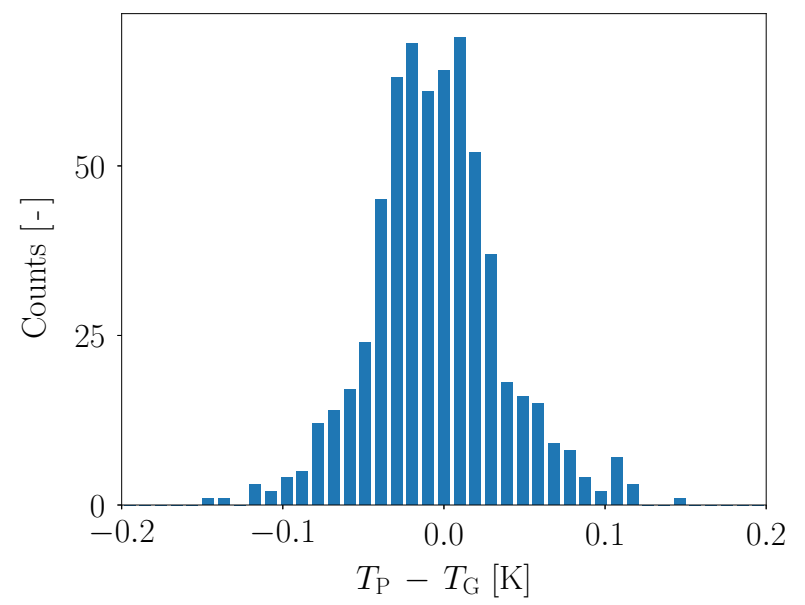

(b)

Figure 6: (a) Deviation field depicting the deviation $T_{\mathrm{P}}-T_{\mathrm{G}}$ of the temperature field depicted in 3 . (b) Histogram of the deviation $T_{\mathrm{P}}-T_{\mathrm{G}}$. The Gaussian shape indicates a normal distribution of the deviations.

and almost randomly distributed across the field of interest. Furthermore, the histogram shows that the vast majority of the deviation is in the range of $-0.1 \mathrm{~K}$ to $0.1 \mathrm{~K}$, which is comparable to the results reported in the literature by Moller et al. (2019). The histogram in Figure 6(b), which almost follows a Gaussian shape, also indicates a normal distribution of the deviations.

In addition to the randomly sampled temperature distribution, the $\mathrm{NN}$ was tested on a stable thermal stratification. Therefore, the top plate of the cell was heated to $\approx 20.9^{\circ} \mathrm{C}$, while the bottom plate was cooled to $\approx 18.3{ }^{\circ} \mathrm{C}$. Thereby, fluid motion is inhibited, and heat is only transferred from the top plate to the bottom plate by means of conduction. As a result, a well-known linear temperature profile along the heights of the cell can be achieved. In addition to the well-known profile, the stratification is temporally stable, compensating the relatively long acquisition time of $\approx 4$ seconds for a full set. The predicted temperature field can be seen in Figure 7(a). The plot shows the smooth transition from the hot temperature at the top to cold temperatures at the bottom. At this point, it is important to mention that the perspective distortion present in the image due to the oblique observation angle is not compensated. This is noticeable in the slight change of the temperature gradient along the $x$-axis and.

For quantitative analysis, the vertical temperature profile at the position $X=0.5$ was extracted and is shown in Figure $7(\mathrm{~b})$. In this Figure, the predicted temperature $T_{\mathrm{P}}$ (solid, black line), the theoretical temperature of the linear profile $T_{\text {lin }}$ (dotted, black line) and the deviation $T_{\mathrm{P}}-T_{\text {lin }}$ are plotted over the vertical position $Y$. To determine the temperature of the linear profile $T_{\text {lin }}$, the temperatures measured by the sensors in the plates were scaled according to the position of the region of interest. By comparing the curves for $T_{\mathrm{P}}$ and $T_{\text {lin }}$, it gets apparent that the linear profile is predicted accurately by the network, especially at the bottom and top of the region of interest. But by viewing the deviation $T_{\mathrm{P}}-T_{\text {lin }}$, a slight overestimation of the temperature profile with a maximum deviation of $\approx 0.15 \mathrm{~K}$ can be seen. However, this overestimation might also be traced back to an imperfect stratification or errors introduced by the scaling. Nevertheless, the profiles show that multi-spectral imaging combined with a neural network for data processing is a promising approach for Particle Image Thermometry that should be further investigated. 


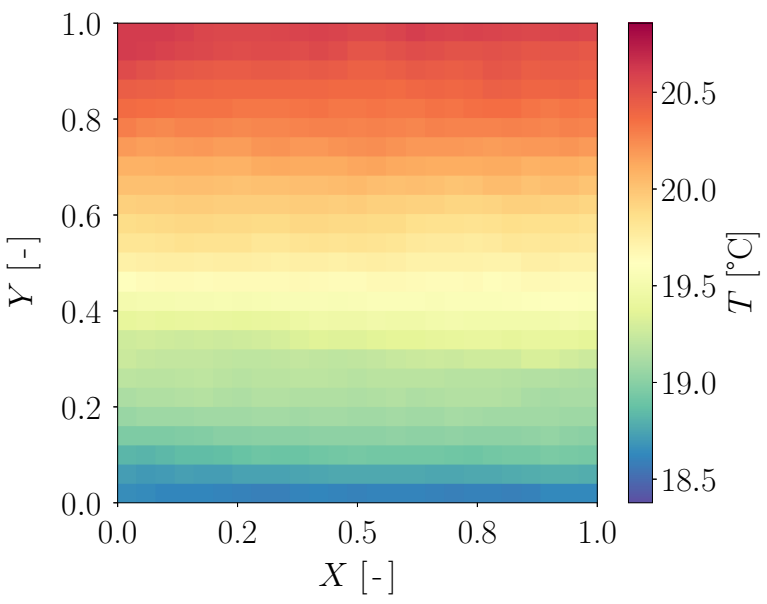

(a)

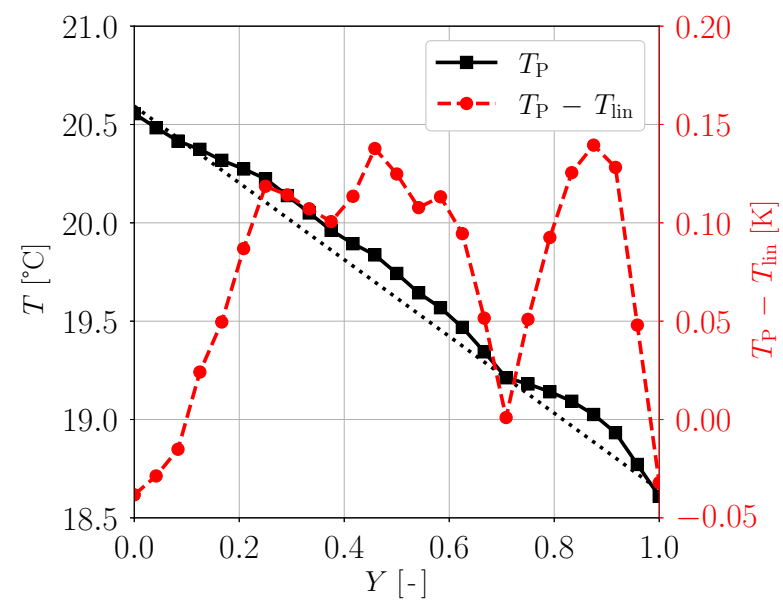

(b)

Figure 7: (a) Temperature field of the stable thermal stratification. (b) Plot of the vertical temperature profile (solid, black line), the theoretical temperature of the linear profile (dotted, black line) and the deviation $T_{\mathrm{P}}-T_{\mathrm{G}}$ over the vertical position $Y$ at $X=0.5$.

\section{Conclusions}

In this work, the feasibility of multi-spectral imaging for Thermochromic Liquid Crystal based Particle Image Thermometry was investigated. An experiment consisting of a transparent, cylindrical cell with adjustable temperature at the bottom and top side was set up. For the acquisition, a multi-spectral camera with twelve color channels was used, and a neural network was trained to predict the temperature from the intensity data. The suitability of the neural network was tested on a randomly sampled temperature distribution and a stable thermal stratification. The test on the randomly sampled temperature distribution showed that the predominant part of the deviations is below $0.1 \mathrm{~K}$. The comparison between the predicted and the expected profile of the stable thermal stratification shows a maximum deviation of $\approx 0.15 \mathrm{~K}$. Both results demonstrate that multi-spectral imaging for PIT combined with neural networks for data processing is promising and should be further investigated. Therefore, the following improvements should be considered: At first, an illumination source with an extended wavelength spectrum at the edge between the visible wavelength range and the near-infrared wavelength range should be utilized. Secondly, the illumination power of the light source should be increased to reduce the exposure time in order to speed up the time required for the recording of a full set of filters. The usage of a super-continuum laser can accomplish both, see König et al. (2019). Alternatively, a camera that simultaneously records all color channels can be employed.

\section{Acknowledgements}

The authors are grateful to Andrei Golomoz, Axel Sichard and Richard Fütterer for their help with the camera configuration and the camera control.

\section{References}

Abram C, Fond B, and Beyrau F (2018) Temperature measurement techniques for gas and liquid flows using thermographic phosphor tracer particles. Progress in Energy and Combustion Science 64:93-156

Anders S, Noto D, Tasaka Y, and Eckert S (2020) Simultaneous optical measurement of temperature and velocity fields in solidifying liquids. Experiments in Fluids 61:1-19

Dabiri D and Gharib M (1991) Digital particle image thermometry: The method and implementation. Experiments in Fluids 11:77-86 
Funatani S, Fujisawa N, and Ikeda H (2004) Simultaneous measurement of temperature and velocity using two-colour LIF combined with PIV with a colour CCD camera and its application to the turbulent buoyant plume. Measurement Science and Technology 15:983

König J, Moller S, Granzow N, and Cierpka C (2019) On the application of a supercontinuum white light laser for simultaneous measurements of temperature and velocity fields using thermochromic liquid crystals. Experimental Thermal and Fluid Science 109:109914

Massing J, Kaden D, Kähler C, and Cierpka C (2016) Luminescent two-color tracer particles for simultaneous velocity and temperature measurements in microfluidics. Measurement Science and Technology 27:115301

Moller S, König J, Resagk C, and Cierpka C (2019) Influence of the illumination spectrum and observation angle on temperature measurements using thermochromic liquid crystals. Measurement Science and Technology 30:084006

Moller S, Resagk C, and Cierpka C (2021) Long-time experimental investigation of turbulent superstructures in Rayleigh-Bénard convection by noninvasive simultaneous measurements of temperature and velocity fields. Experiments in Fluids 62:1-18

Pedregosa F, Varoquaux G, Gramfort A, Michel V, Thirion B, Grisel O, Blondel M, Prettenhofer P, Weiss R, Dubourg V, Vanderplas J, Passos A, Cournapeau D, Brucher M, Perrot M, and Duchesnay E (2011) Scikit-learn: Machine learning in Python. Journal of Machine Learning Research 12:2825-2830

Rosenberger M and Celestre R (2016) Smart multispectral imager for industrial applications. in 2016 IEEE International Conference on Imaging Systems and Techniques (IST). pages 7-12. IEEE 